\title{
ON CERTAIN EQUATION RELATED TO DERIVATIONS ON STANDARD OPERATOR ALGEBRAS AND SEMIPRIME RINGS
}

\author{
IRENA KosI-ULBL \\ University of Maribor, Slovenia
}

\begin{abstract}
In this paper we prove the following result, which is related to a classical result of Chernoff. Let $X$ be a real or complex Banach space, let $\mathcal{A}(X)$ be a standard operator algebra on $X$ and let $\mathcal{L}(X)$ be an algebra of all bounded linear operators on $X$. Suppose we have a linear mapping $D: \mathcal{A}(X) \rightarrow \mathcal{L}(X)$ satisfying the relation $D\left(A^{m+n}\right)=D\left(A^{m}\right) A^{n}+A^{m} D\left(A^{n}\right)$ for all $A \in \mathcal{A}(X)$ and some fixed integers $m \geq 1, n \geq 1$. In this case there exists $B \in \mathcal{L}(X)$, such that $D(A)=A B-B A$ holds for all $A \in \mathcal{F}(X)$, where $\mathcal{F}(X)$ denotes the ideal of all finite rank operators in $\mathcal{L}(X)$. Besides, $D\left(A^{m}\right)=A^{m} B-B A^{m}$ is fulfilled for all $A \in \mathcal{A}(X)$.
\end{abstract}

Throughout, $R$ will represent an associative ring with center $Z(R)$. Given an integer $n>1$, a ring $R$ is said to be $n$-torsion free, if $n x=0, x \in R$ implies $x=0$. Recall that a ring $R$ is prime if for $a, b \in R, a R b=(0)$ implies that either $a=0$ or $b=0$, and is semiprime in case $a R a=(0)$ implies $a=0$. Let $A$ be an algebra over the real or complex field and let $B$ be a subalgebra of $A$. A linear mapping $D: B \rightarrow A$ is called a linear derivation in case $D(x y)=D(x) y+x D(y)$ holds for all pairs $x, y \in B$. In case we have a ring $R$ an additive mapping $D: R \rightarrow R$ is called a derivation if $D(x y)=D(x) y+x D(y)$ holds for all pairs $x, y \in R$ and is called a Jordan derivation in case $D\left(x^{2}\right)=D(x) x+x D(x)$ is fulfilled for all $x \in R$. A derivation $D$ is inner in case there exists $a \in R$, such that $D(x)=a x-x a$ holds for all $x \in R$. Every derivation is a Jordan derivation. The converse is in general not true. A classical result of Herstein ([5]) asserts that any Jordan derivation on a 2 -torsion free prime ring is a derivation. A brief proof

2010 Mathematics Subject Classification. 16N60, 39B05, 46K15.

Key words and phrases. Prime ring, semiprime ring, Banach space, standard operator algebra, derivation, Jordan derivation. 
of Herstein theorem can be found in [1]. Cusack ([4]) generalized Herstein theorem to 2-torsion free semiprime rings (see also [2] for an alternative proof). Let $X$ be a real or complex Banach space and let $\mathcal{L}(X)$ and $\mathcal{F}(X)$ denote the algebra of all bounded linear operators on $X$ and the ideal of all finite rank operators in $\mathcal{L}(X)$, respectively. An algebra $\mathcal{A}(X) \subset \mathcal{L}(X)$ is said to be standard in case $\mathcal{F}(X) \subset \mathcal{A}(X)$. Let us point out that any standard algebra is prime, which is a consequence of Hahn-Banach theorem. $[6,7])$.

Let us start with the following result proved by Chernoff ([3]) (see also

Theorem A. Let $X$ be a real or complex Banach space and let $\mathcal{A}(X)$ be a standard operator algebra. Let $D: \mathcal{A}(X) \rightarrow \mathcal{L}(X)$ be a linear derivation. In this case $D$ is of the form $D(A)=A B-B A$ for all $A \in \mathcal{A}(X)$ and some $B \in \mathcal{L}(X)$.

It is our aim in this paper to prove the following result which is related to the result mentioned above.

TheOREM 1. Let $X$ be a real or complex Banach space and let $\mathcal{A}(X)$ be a standard operator algebra on $X$. Suppose we have a linear mapping $D: \mathcal{A}(X) \rightarrow \mathcal{L}(X)$ satisfying the relation

$$
D\left(A^{m+n}\right)=D\left(A^{m}\right) A^{n}+A^{m} D\left(A^{n}\right)
$$

for all $A \in \mathcal{A}(X)$ and some fixed integers $m \geq 1, n \geq 1$. In this case there exists $B \in \mathcal{L}(X)$, such that the following statements are fulfilled

(i) $D(A)=A B-B A$ for all $A \in \mathcal{F}(X)$,

(ii) $D\left(A^{m}\right)=A^{m} B-B A^{m}$ for all $A \in \mathcal{A}(X)$.

In the proof of the result above we shall use Herstein theorem and Theorem A.

Proof. We have the relation

$$
D\left(A^{m+n}\right)=D\left(A^{m}\right) A^{n}+A^{m} D\left(A^{n}\right) .
$$

Let $A$ be from $\mathcal{F}(X)$ and let $P \in \mathcal{F}(X)$ be a projection with $A P=P A=A$. From the above relation one obtains

$$
D(P)=D(P) P+P D(P) .
$$

Right multiplication of the above relation by $P$ gives

$$
P D(P) P=0 \text {. }
$$


Setting $A+\alpha P$ for $A, \alpha \in \mathcal{F}$, in the relation (1) we obtain

$$
\begin{aligned}
\sum_{i=0}^{m+n}\left(\begin{array}{c}
m+n \\
i
\end{array}\right) D\left(A^{m+n-i}(\alpha P)^{i}\right) & \\
= & D\left(\sum_{i=0}^{m}\left(\begin{array}{c}
m \\
i
\end{array}\right) A^{m-i}(\alpha P)^{i}\right)\left(\sum_{i=0}^{n}\left(\begin{array}{c}
n \\
i
\end{array}\right) A^{n-i}(\alpha P)^{i}\right) \\
& +\left(\sum_{i=0}^{m}\left(\begin{array}{c}
m \\
i
\end{array}\right) A^{m-i}(\alpha P)^{i}\right) D\left(\sum_{i=0}^{n}\left(\begin{array}{c}
n \\
i
\end{array}\right) A^{n-i}(\alpha P)^{i}\right) .
\end{aligned}
$$

Collecting all expressions with coefficient $\alpha^{m+n-2}$ and all expressions with coefficient $\alpha^{m+n-1}$ from the above relation gives

$$
\begin{gathered}
\left(\begin{array}{c}
m+n \\
m+n-2
\end{array}\right) D\left(A^{2}\right)-\left(\begin{array}{c}
m \\
m-2
\end{array}\right)\left(\begin{array}{l}
n \\
n
\end{array}\right) D\left(A^{2}\right) P-\left(\begin{array}{c}
m \\
m-1
\end{array}\right)\left(\begin{array}{c}
n \\
n-1
\end{array}\right) D(A) A \\
-\left(\begin{array}{c}
m \\
m
\end{array}\right)\left(\begin{array}{c}
n \\
n-2
\end{array}\right) D(P) A^{2}-\left(\begin{array}{c}
m \\
m-2
\end{array}\right)\left(\begin{array}{c}
n \\
n
\end{array}\right) A^{2} D(P) \\
-\left(\begin{array}{c}
m \\
m-1
\end{array}\right)\left(\begin{array}{c}
n \\
n-1
\end{array}\right) A D(A)-\left(\begin{array}{c}
m \\
m
\end{array}\right)\left(\begin{array}{c}
n \\
n-2
\end{array}\right) P D\left(A^{2}\right)=0,
\end{gathered}
$$

and

$$
\begin{gathered}
\left(\begin{array}{c}
m+n \\
m+n-1
\end{array}\right) D(A)-\left(\begin{array}{c}
m \\
m-1
\end{array}\right)\left(\begin{array}{l}
n \\
n
\end{array}\right) D(A) P-\left(\begin{array}{c}
m \\
m
\end{array}\right)\left(\begin{array}{c}
n \\
n-1
\end{array}\right) D(P) A \\
-\left(\begin{array}{c}
m \\
m-1
\end{array}\right)\left(\begin{array}{l}
n \\
n
\end{array}\right) A D(P)-\left(\begin{array}{l}
m \\
m
\end{array}\right)\left(\begin{array}{c}
n \\
n-1
\end{array}\right) P D(A)=0,
\end{gathered}
$$

respectively. The above equations reduce into

$$
\begin{aligned}
& (m+n)(m+n-1) D\left(A^{2}\right)=m(m-1) D\left(A^{2}\right) P+n(n-1) P D\left(A^{2}\right) \\
& +2 m n(D(A) A+A D(A))+m(m-1) A^{2} D(P)+n(n-1) D(P) A^{2},
\end{aligned}
$$

and

$$
(m+n) D(A)=m D(A) P+n D(P) A+m A D(P)+n P D(A),
$$

respectively. Applying the relation (2) and the fact that $A P=P A=A$, we have

$$
P D(P) A=(P D(P) P) A=0 .
$$

Similarly one obtains that

$$
A D(P) P=0 .
$$

Right multiplication of the relation (4) by $P$ and using (6) we obtain

$$
D(A) P=D(P) A+P D(A) P .
$$


Similarly one obtains using (5)

$$
P D(A)=A D(P)+P D(A) P .
$$

Subtracting the relation (8) from the relation (7) we obtain

$$
D(A) P-P D(A)-D(P) A+A D(P)=0 .
$$

Using the relation (9) in the relation (4) we obtain

$$
D(A)=D(P) A+P D(A)=D(A) P+A D(P) .
$$

From the above relation one obtains

$$
D\left(A^{2}\right)=D(P) A^{2}+P D\left(A^{2}\right)=D\left(A^{2}\right) P+A^{2} D(P) .
$$

Using the above relation in the relation (3) we obtain after some calculation

$$
D\left(A^{2}\right)=D(A) A+A D(A)
$$

for any $A \in \mathcal{F}(X)$. From the relation (4) one can conclude that $D(A) \in \mathcal{F}(X)$ for any $A \in \mathcal{F}(X)$. It means that $D$ is a mapping which maps $\mathcal{F}(X)$ into itself. According to the relation (12) we have therefore a Jordan derivation on $\mathcal{F}(X)$. As we have mentioned at the beginning of the paper any standard algebra is prime, which is a consequence of Hahn-Banach theorem. Since $\mathcal{F}(X)$ is prime all the assumptions of Herstein theorem are fulfilled and one can conclude that $D$ is a derivation. Applying Theorem A one can conclude that $D$ is of the form

$$
D(A)=A B-B A,
$$

for all $A \in \mathcal{F}(X)$ and some $B \in \mathcal{L}(X)$. This proves the statement $(i)$ of the theorem. It remains to prove the statement $(i i)$. Let us introduce $D_{1}: \mathcal{A}(X) \rightarrow \mathcal{L}(X)$ by $D_{1}(A)=A B-B A$ and consider $D_{0}=D-D_{1}$. The mapping $D_{0}$ is, obviously, linear and satisfies the relation (1). Besides $D_{0}$ vanishes on $\mathcal{F}(X)$. Let $A \in \mathcal{A}(X)$, let $P \in \mathcal{F}(X)$, be a one-dimensional projection and $S=A+P A P-(A P+P A)$. Since, obviously, $S-A \in \mathcal{F}(X)$, we have $D_{0}(S)=D_{0}(A)$. Besides $S P=P S=0$. We have therefore the relation

$$
D_{0}\left(S^{m+n}\right)=D_{0}\left(S^{m}\right) S^{n}+S^{m} D_{0}\left(S^{n}\right) .
$$

Applying the relation (14) and the fact that $S P=P S=0, D_{0}(P)=0$ we obtain

$$
\begin{aligned}
& D_{0}\left(S^{m}\right) S^{n}+S^{m} D_{0}\left(S^{n}\right)=D_{0}\left(S^{m+n}\right)=D_{0}\left(S^{m+n}+P\right) \\
& \quad=D_{0}\left((S+P)^{m+n}\right)=D_{0}\left((S+P)^{m}\right)(S+P)^{n}+(S+P)^{m} D_{0}\left((S+P)^{n}\right) \\
& \quad=D_{0}\left(\left(S^{m}+P\right)\left(S^{n}+P\right)+\left(S^{m}+P\right) D_{0}\left(S^{n}+P\right)\right. \\
& =D_{0}\left(S^{m}\right) S^{n}+D_{0}\left(S^{m}\right) P+S^{m} D_{0}\left(S^{n}\right)+P D_{0}\left(S^{n}\right) .
\end{aligned}
$$

We have therefore

$$
D_{0}\left(A^{m}\right) P+P D_{0}\left(A^{n}\right)=0 .
$$


Putting $2 A$ for $A$ in the above relation one obtains

$$
2^{m-n} D_{0}\left(A^{m}\right) P+P D_{0}\left(A^{n}\right)=0 .
$$

Combining the relations (15) and (16) we arrive at

$$
D_{0}\left(A^{m}\right) P=0 \text {. }
$$

Since $P$ is an arbitrary one-dimensional projection, it follows from the above relation that $D_{0}\left(A^{m}\right)=0$ for any $A \in \mathcal{A}(X)$. Thus we have

$$
0=D_{0}\left(A^{m}\right)=D\left(A^{m}\right)-D_{1}\left(A^{m}\right) \text {, }
$$

which means that

$$
D\left(A^{m}\right)=D_{1}\left(A^{m}\right)=A^{m} B-B A^{m},
$$

for all $A \in \mathcal{A}(X)$. The proof of the theorem is therefore complete.

We proceed with the following conjecture.

CONJECTURE 2. Let $R$ be a semiprime ring with suitable torsion restrictions and let $D: R \rightarrow R$ be an additive mapping satisfying the relation

$$
D\left(x^{m+n}\right)=D\left(x^{m}\right) x^{n}+x^{m} D\left(x^{n}\right)
$$

for all $x \in R$ and some fixed integers $m \geq 1, n \geq 1$. In this case $D$ is a derivation.

We are going to prove the above conjecture in case a ring has the identity element.

TheOREM 3. Let $m \geq 1, n \geq 1$ be some fixed integers and let $R$ be a $2, m$ and $n$-torsion free semiprime ring with the identity element. Suppose we have an additive mapping $D: R \rightarrow R$ satisfying the relation

$$
D\left(x^{m+n}\right)=D\left(x^{m}\right) x^{n}+x^{m} D\left(x^{n}\right)
$$

for all $x \in R$. In this case $D$ is a derivation.

Proof. We have the relation

$$
D\left(x^{m+n}\right)=D\left(x^{m}\right) x^{n}+x^{m} D\left(x^{n}\right), \quad x \in R .
$$

From the above relation it follows immediately that $D(e)=0$, where $e$ stands for the identity element. With the same approach as in the proof of Theorem 1 we obtain from the relation (17)

$$
\begin{gathered}
\left(\begin{array}{c}
m+n \\
m+n-2
\end{array}\right) D\left(x^{2}\right)=\left(\begin{array}{c}
m \\
m-2
\end{array}\right)\left(\begin{array}{l}
n \\
n
\end{array}\right) D\left(x^{2}\right)+\left(\begin{array}{c}
m \\
m-1
\end{array}\right)\left(\begin{array}{c}
n \\
n-1
\end{array}\right) D(x) x \\
+\left(\begin{array}{c}
m \\
m-1
\end{array}\right)\left(\begin{array}{c}
n \\
n-1
\end{array}\right) x D(x)+\left(\begin{array}{c}
m \\
m
\end{array}\right)\left(\begin{array}{c}
n \\
n-2
\end{array}\right) D\left(x^{2}\right), \quad x \in R .
\end{gathered}
$$

From the above relation we obtain after some calculation

$$
D\left(x^{2}\right)=D(x) x+x D(x), \quad x \in R .
$$


In the procedure mentioned above we used the fact that $R$ is $2, m$ and $n$-torsion free. The relation (18) means that $D$ is a Jordan derivation. According to Cusack's generalization of Herstein theorem $D$ is a derivation. The proof of the theorem is complete.

\section{REFERENCES}

[1] M. Brešar and J. Vukman, Jordan derivations on prime rings, Bull. Austral. Math. Soc. 37 (1988), 321-322.

[2] M. Brešar, Jordan derivations on semiprime rings, Proc. Amer. Math. Soc. 104 (1988), 1003-1006.

[3] P. R. Chernoff, Representations, automorphisms, and derivations of some operator algebras, J. Functional Analysis 12 (1973), 275-289.

[4] J. M. Cusack, Jordan derivations on rings, Proc. Amer. Math. Soc. 53 (1975), 321-324.

[5] I. N. Herstein, Jordan derivations of prime rings, Proc. Amer. Math. Soc. 8 (1957), $1104-1110$

[6] N. Širovnik and J. Vukman, On functional equation related to derivations and bicircular projections, Oper. Matrices 8 (2014), 849-860.

[7] J. Vukman, On automorphisms and derivations of operator algebras, Glas. Mat. Ser. III 19(39) (1984), 135-138.

I. Kosi-Ulbl

Faculty of Mechanical Engineering

University of Maribor

Smetanova 17, 2000 Maribor

Slovenia

E-mail: irena.kosi@um.si

Received: 23.11.2016.

Revised: 15.3.2017. 